\title{
Índice cronotrópico-metabólico na doença de Chagas
}

\author{
Chronotropic-metabolic index in Chagas' disease
}

\author{
Ana Luiza Lunardi Rocha ${ }^{1}$, Manoel Otávio da Costa Rocha ${ }^{1}$, Bruno Otávio Soares Teixeira ${ }^{3}$, \\ Federico Lombardi, Cláudia Drumond Guimarães Abreu², Roberto José Bittencourt ${ }^{1}$, \\ Márcio Vinícius Lins Barros ${ }^{1,5}$ e Antonio Luiz Pinho Ribeiro ${ }^{1,2}$
}

\begin{abstract}
RESUMO
A insuficiência cronotrópica constitui achado comum entre os pacientes chagásicos. Novas metodologias estão sendo empregadas na avaliação da resposta cronotrópica em vários grupos de pacientes. 0 índice cronotrópico-metabólico, um desses novos métodos, quantifica a relação entre 0 aumento da freqüência cardíaca e 0 consumo máximo de oxigênio $\left(\mathrm{VO}_{2} \max \right)$ durante 0 teste ergométrico. A resposta normal é linear, com índice em torno de 1,0. Objetivamos avaliar a resposta cronotrópica e em indivíduos saudáveis e pacientes chagásicos com e sem disfunção ventricular esquerda, utilizando-se do índice cronotrópico-metabólico. Foram avaliados 171 pacientes com doença de Chagas sem doenças associadas e 24 controles submetidos a protocolo clínico e ao teste ergométrico máximo. Os chagásicos foram divididos em dois grupos: $\mathrm{Ch} 1=$ pacientes com fração de ejeção $(\mathrm{FE})>39 \%$ eCh 2= FE < 40\%. Aanálise e o cálculo do índice cronotrópicometabólico foram feitos pelo método de Wilkoff. Os pacientes chagásicos apresentaram maior idade e maior prevalência de bloqueio completo de ramo direito, assim como menor $\mathrm{VO}_{2}$ max ao teste ergométrico. Ambos os grupos de chagásicos

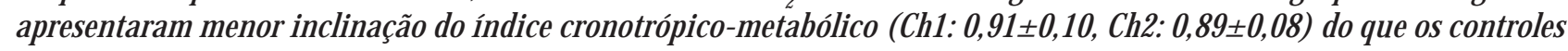
$(1,0 \pm 0,12, p<0,001)$. Pacientes com doença de Chagas com e sem disfunção ventricular esquerda podem apresentar resposta cronotrópica deprimida, manifesta por menor inclinação do índice cronotrópico-metabólico.
\end{abstract}

Palavras-chaves: Doença de Chagas. Sistema nervoso autônomo. Cronotropismo.

\begin{abstract}
Chronotropic incompetence is a common feature in Chagas' disease patients. New methodologies are now available to evaluate the chronotropic response in different subsets. The chronotropic-metabolic index (CM) is one of these new indexes and quantifies the relationship between the increment of heart rate and the maximal oxygen consumption $\left(\mathrm{VO}_{2}\right.$ max $)$ during exercise testing. In normal subjects there is linear response and the index is round 1.0. The aim of the study was to evaluate the chronotropic response in healthy controls and Chagas' disease patients with and without left ventricular dysfunction, using CM. Twenty-four controls and 171 Chagas' disease patients underwent a clinical protocol and maximal exercise testing. Chagas' disease patients were divided into two groups: $\mathrm{Ch} 1=$ patients with ejection fraction $(\mathrm{EF})>39 \%$ and $\mathrm{Ch} 2=\mathrm{EF}<40 \%$. CM was analyzed and calculated according to the Wilkoff method. Chagas' disease patients were older than controls and showed higher prevalence of right bundle branch block, as well as lower $\mathrm{VO}_{2}$ max during exercise. Both groups of Chagas' disease patients showed a less steep curve in the chronotropic-metabolic index (Ch1: 0.91 \pm 0.10 , Ch2: 0.89 \pm 0.08 ) than controls $(1.0 \pm 0.12, \mathrm{p}<0.001)$. Chagas' disease patients with and without left ventricular dysfunction chronotropic incompetence may exhibit reduced chronotropic response to exercise, expressed by a less steep chronotropic-metabolic index.
\end{abstract}

Key-words: Chagas' disease. Autonomic nervous system. Chronotropism.

\footnotetext{
1. Pós-Graduação em Ciências da Saúde: Infectologia e Medicina Tropical da Faculdade de Medicina da Universidade de Minas Gerais, Belo Horizonte, MG. 2. Serviço de Cardiologia da Hospital das Clínicas da Universidade Federal of Minas Gerais, Belo Horizonte, MG. 3. Laboratório de Modelagem, Análise e Controle de Sistemas Năo-Lineares ( MACSIN) do Departamento de Engenharia Eletrônica da Universidade Federal de Minas Gerais, Belo Horizonte, MG. 4. Cardiologia, Ospedale San Paolo, Dipartimento di Medicina, Chirurgia e Odontoiatria, Università di Milano, Milão, Itália. 5. Ecoar - Medicina Diagnóstica, Belo Horizonte, MG, Brasil.

Financiado por auxílios do Conselho Nacional do Desenvolvimento Científico e Tecnológico (CNPq), Fundação de Amparo à Pesquisa do Estado de Minas Gerais ( FAPEMIG), Coordenadoria de Aperfeiçoamento do Ensino Superior (CAPES) e Consiglio Nazionale delle Richerche (CNR), Itália.

Endereço para correspondência: Dr. Antonio L.P. Ribeiro. R. Campanha 98/101, 30310-770 Belo Horizonte, MG, Brasil.

Tel: 5531 3287-9213; Fax: 5531 3284-7298

e-mail: tom@ hc.ufmg.br

Recebido para publicação em 20/2/2004

Aceito em 27/6/2005
} 
A doença de Chagas representa uma das mais importantes causas de morte na América Latina, onde quase 20 milhões de pessoas estão infectadas ${ }^{15}$. A doença causa grande impacto socioeconômico já que acomete muitos indivíduos em idade produtiva. 0 curso clínico da doença é bastante variável. Enquanto muitos indivíduos podem desenvolver cardiopatia grave, às vezes fatal, outros permanecem totalmente assintomáticos sem nunca desenvolverem formas crônicas. Sendo assim, torna-se importante definir marcadores de risco de evolução desfavorável e de morte nos pacientes com doença de Chagas ${ }^{1}$.

A insuficiência cronotrópica (ICr) é caracterizada como a incapacidade de aumentar a frequêencia cardíaca ( FC) durante 0 teste ergométrico ( TE) e está claramente relacionada com 0 pior prognóstico dos pacientes com coronariopatia ${ }^{8}$. Embora a ICr seja um evento relativamente comum em pacientes chagásicos, sua importância na evolução destes é ainda pouco conhecida $^{5} 6912$. Novas metodologias estão sendo empregadas na avaliação da resposta cronotrópica em vários grupos de pacientes. 0 índice cronotrópico-metabólico (ICrM) , um desses novos métodos, é obtido pela análise da relação entre 0 aumento da frequêencia cardíaca ( $\mathrm{FC}$ ) e 0 consumo máximo de oxigênio (V02) durante 0 teste ergométrico (TE) ${ }^{314}$. A resposta normal é linear, com índice de 1,0. Existem vantagens teóricas na avaliação da ICr pelo ICrM, já que este avalia a ICr em relação ao consumo de oxigênio, mas o seu comportamento na doença de Chagas é ainda desconhecido.

Este estudo tem como objetivo avaliar a resposta cronotrópica, utilizando-se do ICrM, em pacientes chagásicos com e sem disfunção ventricular esquerda (dVE) comparandoos com pacientes controles, sem doença de Chagas.

\section{PACIENTES E MÉTODOS}

0 estudo, de desenho clínico epidemiológico transversal, faz parte de estudo prospectivo intitulado "Disfunção autonômica na doença de Chagas: mecanismos eimplicações prognósticas" e foi aprovado pelo Comitê de Ética em Pesquisa da UFMG. Pacientes foram examinados no Ambulatório de Referência em Doença de Chagas do Centro de Treinamento e Referência em Doenças Infecciosas e Parasitárias da Universidade Federal de Minas Gerais. 0 diagnóstico de doença de Chagas foi confirmado por sorologias positivas para T. cruzi por dois ou mais métodos diferentes (ELISA, RIFI, hemaglutinação indireta) . Pacientes não selecionados, atendidos consecutivamente no Ambulatório de Referência no período entre janeiro de 1998 e julho de 1999 e que concordaram em participar do estudo, assinaram 0 termo de consentimento e foram submetidos a anamnese, exame físico, ECG, exames laboratoriais e radiografia de tórax. Os critérios de exclusão foram: 1) presença de outra doença sistêmica, como cardiopatia, diabetes, disfunção tireoideana, doença pulmonar obstrutiva crônica, insuficiência hepática ou renal, anemia, hipertensão arterial; 2) gravidez; 3) alcoolismo; 4) uso de drogas que comprometem a resposta cronotrópica ao TE; 5) interrupção do TE por indicação médica (dor torácica, tonteira, arritmia, aumento exagerado da pressão arterial). Foram avaliados 24 indivíduos saudáveis e 171 pacientes com doença de Chagas. Os chagásicos foram divididos em dois grupos: Ch1: pacientes com fração de ejeção (FE) $\geq 40 \%$ e Ch2: pacientes com FE $<40 \%$. Todos foram submetidos ao protocolo clínico, TE e ecocardiograma.

Os participantes do estudo foram submetidos ao ecodopplercardiograma colorido utilizando um equipamento HDI 5000. As medidas foram realizadas por um experiente ecocardiografista (MVB) de acordo com as recomendações da Sociedade Americana de Ecocardiografia ${ }^{13}$ A fração de ejeção do ventrículo esquerdo foi obtida pelo método de Simpson ${ }^{13}$.

0 TE foi realizado de acordo com o protocolo de Bruce, em esteira rolante. Os pacientes foram encorajados a se exercitar até atingir 0 pico da FC. A resposta cronotrópica foi avaliada pela porcentagem atingida da FC máxima prevista para idade, de acordo com a fórmula de Astrand (Fcmáx=220- idade). A análise e 0 cálculo do ICrM foram feitos pelo método de Wilkoff ${ }^{14}$ que avalia a variação da freqüência cardíaca com função linear do consumo de oxigênio ao esforço. Os indivíduos normais exibem uma resposta linear na porcentagem da FC em relação à porcentagem da reserva metabólica, com valores próximos de um, enquanto a insuficiência cronotrópica se manifesta por valores mais baixos.

Os dados foram analisados utilizando-se os softwares Epiinfo versão 6 e SPSS versão 10. Variáveis quantitativas foram descritas pela média ou mediana e medidas de dispersão (desvio padrão e intervalo interquartil, respectivamente). A análise da normalidade e da homogeneidade da variância foi realizada antes da utilização de métodos paramétricos; transformações matemáticas foram realizadas se convenientes. Análises de variância (ANOVA) e de co-variância (ANCOVA, com ajuste para a idade) foram utilizadas para comparação entre os grupos, com comparação de médias realizada com correção de Bonferroni para comparações múltiplas. Coeficientes de correlação entre 0 ICrM e outras variáveis quantitativas foram calculados pelos métodos de Pearson (r) e de Spearmann (rs), dependendo da distribuição normal ou não das variáveis. Variáveis qualitativas foram descritas pela frequêencia e proporção e analisadas em tabelas $2 \mathrm{x} \mathrm{k}$ pelo teste do qui-quadrado. Em todos os testes, alfa (p) foi considerado <0,05 como um indicador de significância estatística.

\section{RESULTADOS}

Pacientes chagásicos apresentaram maior idade e maior prevalência de bloqueio completo de ramo direito, enquanto chagásicos com depressão da FEapresentaram maior prevalência de disfunção diastólica e aneurisma de VE (Tabela 1). Ambos os grupos de chagásicos apresentaram menor inclinação do ICrM como menor porcentagem da freqüência cardíaca máxima atingida ao esforço, além de tendência a menor consumo máximo de oxigênio (Tabela 2). 0 ICrM não se correlacionou com a FEVE: rs $=0,05, p=0,48$ (Figura 1). 0 ICrM apresentou elevada correlação com a porcentagem atingida da freqüência cardíaca 
Tabela 1 - Características gerais e ao ecocardiograma de indivíduos saudáveis (controles) e chagásicos com FE maior ou igual (Ch1) ou menor que $40 \%$ (Ch2).

\begin{tabular}{lccccc}
\hline & $\begin{array}{c}\text { Controle } \\
\mathrm{n}=24\end{array}$ & $\mathrm{Ch} 1$ & $\mathrm{Ch} 2$ & & \\
& $\mathrm{n}=154$ & $\mathrm{n}=17$ & $\mathrm{p}$ & \\
\hline Idade (anos) & $35,6 \pm 9,3$ & $41,7 \pm 9,3$ & $42,8 \pm 9,2$ & 0,01 & $0<1=2$ \\
Mulheres $(\%)^{*}$ & 29 & 43 & 41 &, 415 & \\
BRD $(\%)^{*}$ & 0 & 25,5 & 43,9 & $<, 001$ & $0<1=2$ \\
Fração de ejeção VE $(\%) \dagger$ & $64(61-66)$ & $62(58-65)$ & $35(31-39)$ & $<, 001$ & $0=1>2$ \\
Disfunção diastólica $(\%)^{*}$ & 0 & 5,8 & 51,3 & $<, 001$ & $0<1<2$ \\
Aneurisma de VE $(\%)^{*}$ & 0 & 15,6 & 52,9 & $<, 001$ & $0<1<2$ \\
\hline
\end{tabular}

Valores expressos em médias \pm DP quando apropriado, exceto *, expresso em proporções e $\dagger$, como medianas (intervalo interquartil). $\mathrm{BRD}=$ bloqueio completo de ramo direito, $\mathrm{VE}=$ ventrículo esquerdo.

Tabela 2 - Características ao teste ergométrico de indivíduos saudáveis (controles) e chagásicos com FE maior ou igual (Ch1) ou menor que $40 \%$ (Ch2).

\begin{tabular}{|c|c|c|c|c|c|}
\hline & $\begin{array}{c}\text { Controle } \\
\mathrm{n}=24\end{array}$ & $\begin{array}{c}\mathrm{Ch} 1 \\
\mathrm{n}=154\end{array}$ & $\begin{array}{c}\text { Ch } 2 \\
n=17\end{array}$ & $\mathrm{p}$ & \\
\hline Duração de exercício (s) † \# & 844 (780-1001) & $719,5(612-861)$ & $649(620-792)$ & 113 & \\
\hline VO-- ${ }_{2} \max (\mathrm{ml} / \mathrm{kg} / \mathrm{min})^{\#}$ & $49,4(10,1)$ & $44,7(9,4)$ & $44,1(8,3)$ & 058 & \\
\hline FC basal (bpm) & $71 \pm 13$ & $69 \pm 12$ & $71 \pm 12$ & 614 & \\
\hline FC máxima (bpm) † \# & $188,5(181-196)$ & $164,5(152-176)$ & $160(143-173)$ & $<0,001$ & $0>1=2$ \\
\hline$\Delta \mathrm{FC}(\mathrm{bpm})$ & $112(13)$ & $94(19)$ & $86(19)$ & $<0,001$ & $0>1=2$ \\
\hline FC máxima atingida $(\%)^{\dagger}$ & $101(98-104)$ & $92(87-98)$ & $89(83-101)$ & $<0,001$ & $0>1=2$ \\
\hline $\mathrm{ICrM}^{\#}$ & $1,00(0,12)$ & $0,91(0,10)$ & $0,89(0,08)$ & $<0,001$ & $0>1=2$ \\
\hline
\end{tabular}

Valores expressos em médias \pm DP quando apropriado, exceto *, expresso em proporçoes e $\dagger$, como medianas (intervalo interquartil). S: segundos; $\mathrm{VO}_{2}$ max: consumo de oxigênio máximo estimado. FC: frequiência cardíaca; bpm: batimentos por minuto; $\triangle \mathrm{HR}$ : diferença entre a FC de pico do esforço e basal. ICrM: Inclinação obtida pelo gráfico da variação da frequêencia cardíaca ao esforço sobre a reserva metabólica. Os valores de p se referem a análise ANOVA ou ANCOVA ( ajustados para idade\#) para os índices, com comparação de médias pelo método de Bonferroni.

prevista para a idade $(r=0,60, p<0,001$, Figura 2$)$ e 0 consumo máximo de oxigênio estimado ( $\mathrm{rs}=0,344, \mathrm{P}<0,001$ ). A diferença entre 0 ICrM obtido entre os controles e chagásicos dos dois grupos persiste significativa $(p=0,002)$ mesmo após ajuste por análise de covariância para a idade e 0 consumo máximo de oxigênio estimado (controle $\mathrm{x}$ Ch1 $\mathrm{p}=0,002$, controle $x$ Ch2 $p=0,018$, Ch1 $x$ Ch2 $p=1,000)$.

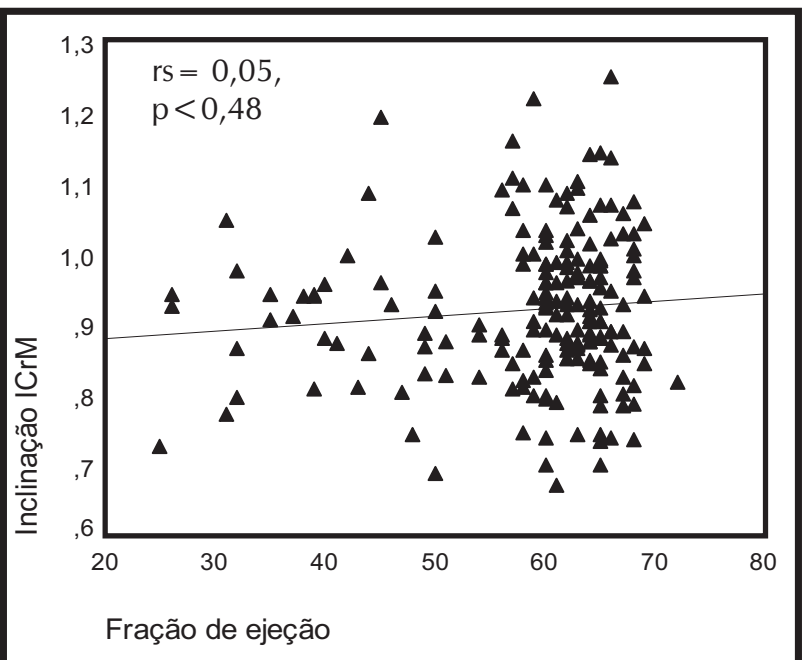

Figura 1 - Correlação entre a fração de ejeção do ventrículo esquerdo e a resposta cronotrópica ao esforço, avaliada pela inclinação do índice cronotrópico-metabólico (ICrM) em 171 chagásicos e 24 controles.

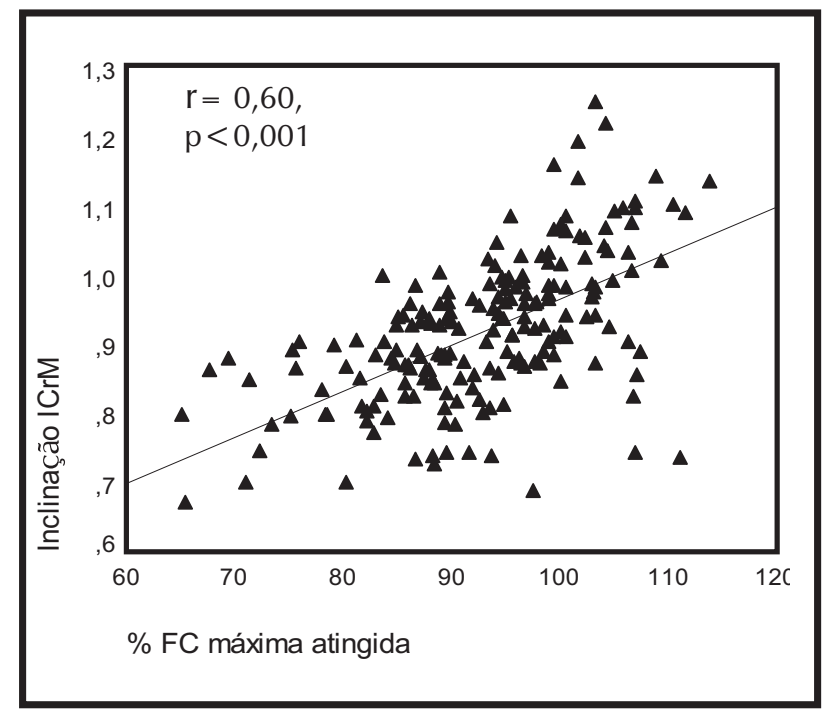

Figura 2 - Correlação entre a porcentagem atingida da freqüência cardíaca prevista para a idade e o índice cronotrópico-metabólico (ICrM) em 171 chagásicos e 24 controles.

\section{DISCUSSÃ0}

A regulação da freqüência cardíaca pode estar prejudicada em diversas situações patológicas, sabendo-se que o pico da frequêencia cardíaca nos pacientes com coronariopatia é menor do que nas pessoas sadias, ao exercício máximo $0^{4}$. Mais recentemente, Lauer e colaboradores encontraram que a insuficiência cronotrópica associa-se com aumento da 
mortalidade total e do risco de doença arterial coronariana ${ }^{8}$. Adicionalmente, em uma coorte de indivíduos assintomáticos, a presença de insuficiência cronotrópica foi previsível pelo aumento do tamanho da cavidade ventricular esquerda e, nos homens, pela depressão da fração de ejeção do ventrículo esquerdo ${ }^{7}$. Neste estudo, o menor aumento da frequêencia cardíaca ao esforço foi observado em ambos os grupos de pacientes chagásicos, de forma independente da presença de disfunção ventricular esquerda. A insuficiência cronotrópica não é incomum na fase crônica da doença de Chagas que, embora de impacto prognóstico incerto, tem sido atribuída à disfunção autonômica predominantemente vagal ou à disfunção do nó sinusal ${ }^{256}$.

Na verdade, diversos estudos têm demonstrado distúrbio precoce da função autonômica, em graus variáveis, em diferentes formas clínicas da doença de Chagas, com potencial papel na fisiopatogênese da morte súbita em pacientes chagásicos ${ }^{1011}$. Assim, é possível que a insuficiência cronotrópica observada ao teste ergométrico em pacientes com doença de Chagas sem disfunção ventricular esquerda significativa sejam um marcador de disfunção autonômica precoce observada nos estudos supracitados.

Os achados do presente trabalho confirmam observações prévias de Gallo e cols ${ }^{56}$ demonstrando um menor aumento da freqüência cardíaca em pacientes com doença de Chagas, usando protocolos experimentais tanto em esteira como em cicloergômetro, correlacionando 0 achado à redução do controle autonômico vagal. Nossos resultados diferem dos estudos precedentes pelo uso de protocolo rotineiro na prática clínica (Bruce) em estudo transversal realizado em amostra representativa da população ambulatorial com a doença de Chagas.

Embora, teoricamente, o ICrM seja método mais sensível e fidedigno para avaliação da insuficiência cronotrópica, nós encontramos excelente correlação entre este novo método e a avaliação convencional na amostra em questão. Como a inclinação da reta não é o único parâmetro que pode ser analisado pelo método de Wilkoff, análises adicionais são necessárias para se avaliar se essa nova metodologia tem algum papel na avaliação da resposta ao esforço no paciente chagásico.

Algumas limitações do presente estudo merecem discussão. A resposta cronotrópica está significativamente relacionada ao consumo máximo estimado de oxigênio, embora não possamos afirmar, a partir dos dados do presente estudo, se éa insuficiência cronotrópica que provoca uma menor capacidade aeróbica ou vice-versa. Entretanto, chagásicos apresentam menor ICrM mesmo após 0 ajuste para 0 consumo máximo estimado de oxigênio, 0 que comprova que a menor capacidade aeróbica não é a principal causa da insuficiência cronotrópica observada entre os chagásicos. Uma limitação adicional se relaciona ao fato de que 0 cálculo indireto do consumo máximo de oxigênio é uma medida relativamente imprecisa, que não substitui a medida direta, não realizada neste estudo. Entretanto, os objetivos do presente estudo estão voltados ao estudo do ICrM e as considerações acerca da capacidade aeróbica são secundárias aos resultados principais.

Concluindo, a insuficiência cronotrópica está presente em pacientes chagásicos independentemente da presença de disfunção ventricular esquerda. 0 ICrM pode ser aplicado no estudo da resposta ao esforço em pacientes chagásicos, embora a insuficiência cronotrópica possa ser reconhecida, de forma mais simples, pela avaliação da porcentagem da freqüência cardíaca máxima atingida. 0 significado fisiopatológico e 0 impacto prognóstico da ICr na doença de Chagas permanecem obscuros e devem ser avaliados em novos estudos.

\section{REFERÊNCIAS BIBLIOGRÁFICAS}

1. Carrasco HA, Parada H, Guerrero L, Duque M, Duran D, Molina C. Prognostic implications of clinical, electrocardiographic and hemodynamic findings in chronic Chagas' disease. International Journal Cardiology 43:2738, 1994.

2. Chiale PA, Ferrari I, Mahler E, Vallazza MA, Elizari MV, Rosenbaum MB, Levin MJ. Differential profile and biochemical effects of antiautonomic membrane receptor antibodies in ventricular arrhythmias and sinus node dysfunction. Circulation 103:1765-1771, 2001.

3. Freedman RA, Hopper DL, Mah J, Hummel J, Wilkoff BL. Assessment of pacemaker chronotropic response: implementation of the Wilkoff mathematical model. Pacing Clinical Electrophysiology 24:1748-1754, 2001.

4. Froelicher VF, Myers J. Exercise and the Heart. $4^{\text {th }}$ edition. WB Saunders, Philadelphia, 2000.

5. Gallo Jr L, A Neto J, Manco JC, Rassi A, Amorim DS. Abnormal heart rate responses during exercise in patients with Chagas' disease. Cardiology 60:147-162, 1975.

6. Gallo Jr L, Morelo Filho J, Maciel BC, Marin-Neto JA, Martins LE, Lima Filho EC. Functional evaluation of sympathetic and parasympathetic system in Chagas' disease using dynamic exercise. Cardiovascular Research 21:922-927, 1987

7. Lauer MS, Larson MG, Evans JC, Levy D. Association of left ventricular dilatation and hypertrophy with chronotropic incompetence in the Framingham Heart Study. American Heart Journal 137:903-909, 1999.

8. Lauer MS, Okin PM, Larson M, Evans JC, Levy D. Impaired heart rate response to graded exercise. Implications of chronotropic incompetence in the Framingham Heart Study. Circulation 93:1520-1526, 1996.

9. Pereira MH, Brito FS, Ambrose JA, Pereira CB, Levi GC, Neto VA, Martinez EE. Exercise testing in the latent phase of Chagas' disease. Clinical Cardiology 7:261-265, 1984.

10. Ribeiro ALP, Lombardi F, Sousa MR, Barros MVL, Porta A, Barros V, Gomes MED, Machado FS, Rocha MOC. Power-Law Behavior of Heart Rate Variability in Chagas Disease. American Journal of Cardiology 89:414-418, 2002.

11. Ribeiro ALP, Moraes RS, Ribeiro JP, Ferlin EL, Torres RM, Oliveira E, Rocha MO. Parasympathetic dysautonomia precedes left ventricular systolic dysfunction in Chagas disease. American Heart Journal 141:260-265, 2001.

12. Ribeiro ALP, Tostes VTV, Torres RM, Abreu CDG, Couto MOR, Gontijo ECD, Rocha MOC. Teste ergométrico em chagásicos sem cardiopatia aparente. Arquivos Brasileiros de Cardiologia 65:96, 1995.

13. Schiller NB, Shah PM, Crawford M, DeMaria A, Devereux R, Feigenbaum H, Gutgesell H, Reichek N, Sahn D, Schnittger I. Recommendations for quantitation of the left ventricle by two-dimensional echocardiography. American Society of Echocardiography Committee on Standards, Subcommittee on Quantitation of Two-Dimensional Echocardiograms. Journal of the American Society of Echocardiography 2:358-367, 1989.

14. Wilkoff BL, Corey J, Blackburn G. A mathematical model of the cardiac chronotropic response to exercise. Journal of Electrophysiology 3:176180, 1989.

15. World Health Organization. Expert Committee on the Control of Chagas Disease. Control of Chagas Disease 1-95, 1991. 